\title{
Erratum: Full Eulerian lattice Boltzmann model for conjugate heat transfer [Phys. Rev. E 92, 063305 (2015)]
}

Yang Hu, Decai Li, Shi Shu, and Xiaodong Niu

(Received 23 February 2016; published 22 March 2016)

DOI: 10.1103/PhysRevE.93.039902

There are corrections to a few equations in the paper. The terms $\left(y_{j+(1 / 2)}-y_{j-(1 / 2)}\right),\left(x_{i+(1 / 2)}-x_{i-(1 / 2)}\right)$ in Eqs. (31) and (32) should be deleted.

Equation (31) should read

$$
\begin{aligned}
& \int_{x_{i+(1 / 2)}, y_{j-(1 / 2)}}^{x_{i+(1 / 2)}, y_{j+(1 / 2)}} \delta Q(X, Y) D\left(x_{i}-X\right) \delta\left(y_{j}-Y\right) d Y \\
& \quad=\int_{x_{i+(1 / 2)}, y_{j-(1 / 2)}}^{x_{i+(1 / 2)}, y_{j+(1 / 2)}} \delta Q\left(x_{i+(1 / 2)}, Y\right) D\left(x_{i}-x_{i+(1 / 2)}\right) \delta\left(y_{j}-Y\right) d Y \\
& \quad \approx D\left(x_{i}-x_{i+(1 / 2)}\right) \int_{x_{i+(1 / 2)}, y_{j-(1 / 2)}}^{x_{i+(1 / 2)}, y_{j+(1 / 2)}} \delta Q\left(x_{i+(1 / 2)}, Y\right) \delta\left(y_{j}-Y\right) d Y \\
& \quad \approx \delta Q\left(x_{i+(1 / 2)}, y_{j}\right) D\left(x_{i}-x_{i+(1 / 2)}\right) .
\end{aligned}
$$

Equation (32) should read

$$
\begin{aligned}
& \int_{x_{i-(1 / 2)}, y_{j+(1 / 2)}}^{x_{i+(1 / 2)}, y_{j+(1 / 2)}} \delta Q(X, Y) \delta\left(x_{i}-X\right) D\left(y_{j}-Y\right) d X \\
& \quad=\int_{x_{i-(1 / 2)}, y_{j+(1 / 2)}}^{x_{i+(1 / 2)}, y_{j+(1 / 2)}} \delta Q\left(X, y_{j+(1 / 2)}\right) \delta\left(x_{i}-X\right) D\left(y_{j}-y_{j+(1 / 2)}\right) d X \\
& \quad \approx D\left(y_{j}-y_{j+(1 / 2)}\right) \int_{x_{i-(1 / 2)}, y_{j+(1 / 2)}}^{x_{i+(1 / 2)}, y_{j+(1 / 2)}} \delta Q\left(X, y_{j+(1 / 2)}\right) \delta\left(x_{i}-X\right) d X \\
& \quad \approx \delta Q\left(x_{i}, y_{j+(1 / 2)}\right) D\left(y_{j}-y_{j+(1 / 2)}\right) .
\end{aligned}
$$

In Eqs. (37), (38), (39), (40), (41), and (42), some coefficients and brackets must be corrected.

Equation (37) should read

$$
\kappa_{1} \frac{\partial T_{1}}{\partial x}=\kappa_{2} \frac{\partial T_{2}}{\partial x}=\frac{2\left(1-\frac{\sigma_{3}}{2}\right)\left[\left(m_{3}-m_{3}^{\mathrm{eq}}\right)+\frac{\sigma_{4}}{2}\left(m_{4}-m_{4}^{\mathrm{eq}}\right)\right]}{\frac{1}{\left(\rho c_{p}\right)_{1}}+\frac{1}{\left(\rho c_{p}\right)_{2}}} .
$$

Equation (38) should read

$$
\kappa_{1} \frac{\partial T_{1}}{\partial y}=\kappa_{2} \frac{\partial T_{2}}{\partial y}=\frac{2\left(1-\frac{\sigma_{5}}{2}\right)\left[\left(m_{5}-m_{5}^{\mathrm{eq}}\right)+\frac{\sigma_{4}}{2}\left(m_{6}-m_{6}^{\mathrm{eq}}\right)\right]}{\frac{1}{\left(\rho c_{p}\right)_{1}}+\frac{1}{\left(\rho c_{p}\right)_{2}}} .
$$

Equation (39) should read

$$
R_{x}(\mathbf{x})=\left(1-\frac{\sigma_{3}}{2}\right)\left\{\left[m_{3}(\mathbf{x})-m_{3}^{\mathrm{eq}}(\mathbf{x})\right]+\frac{\sigma_{4}}{2}\left[m_{4}(\mathbf{x})-m_{4}^{\mathrm{eq}}(\mathbf{x})\right]\right\} .
$$

Equation (40) should read

$$
R_{y}(\mathbf{x})=\left(1-\frac{\sigma_{5}}{2}\right)\left\{\left[m_{5}(\mathbf{x})-m_{5}^{\mathrm{eq}}(\mathbf{x})\right]+\frac{\sigma_{4}}{2}\left[m_{6}(\mathbf{x})-m_{6}^{\mathrm{eq}}(\mathbf{x})\right]\right\} .
$$

Equation (41) should read

$$
\delta Q\left(x_{i+\frac{1}{2}}, y_{j}\right)=\frac{\left[R_{x}\left(x_{i}, y_{j}\right)+R_{x}\left(x_{i+1}, y_{j}\right)\right]\left(\frac{1}{\left(\rho c_{p}\right)_{1}}-\frac{1}{\left(\rho c_{p}\right)_{2}}\right)}{\frac{1}{\left(\rho c_{p}\right)_{1}}+\frac{1}{\left(\rho c_{p}\right)_{2}}}
$$


Equation (42) should read

$$
\delta Q\left(x_{i}, y_{j+\frac{1}{2}}\right)=\frac{\left[R_{y}\left(x_{i}, y_{j}\right)+R_{y}\left(x_{i}, y_{j+1}\right)\right]\left(\frac{1}{\left(\rho c_{p}\right)_{1}}-\frac{1}{\left(\rho c_{p}\right)_{2}}\right)}{\frac{1}{\left(\rho c_{p}\right)_{1}}+\frac{1}{\left(\rho c_{p}\right)_{2}}} .
$$

Moreover, in Eq. (28), $1 \leqslant r \leqslant 1.5$ should be replaced by $0.5 \leqslant r \leqslant 1.5$. Equation (33) should read

$$
\nabla(\chi T)=\left\{\left(1-\frac{\sigma_{3}}{2}\right)\left[\left(m_{3}-m_{3}^{\mathrm{eq}}\right)+\frac{\sigma_{4}}{2}\left(m_{4}-m_{4}^{\mathrm{eq}}\right)\right],\left(1-\frac{\sigma_{5}}{2}\right)\left[\left(m_{5}-m_{5}^{\mathrm{eq}}\right)+\frac{\sigma_{6}}{2}\left(m_{6}-m_{6}^{\mathrm{eq}}\right)\right]\right\} .
$$

It should be noted that the corrected equations were used in the code implementation. The corrections do not affect the discussion and conclusions.

We are grateful to Mufeng Chen from Shantou University for alerting us to these issues. 